\title{
Thought Experiments and the Ostia of Paranasal Sinuses
}

\author{
Mariano B. Caparas \\ Department of Otorhinolaryngology, College of Medicine and Philippine General Hospital, University of the Philippines Manila
}

\section{Introduction}

It behooves us to understand the power of gravity or its diminished or negative effect as we, Homo sapiens, evolve from going around from four limbs to bipedalism or working mostly erect or sitting on our buttocks. Evolution doesn't favor a particular desire or anticipate the future; its particular end is to get that individual to live long and propagate more efficiently. Evolution cannot give you all the favorable advantages; only that selfish genes will make us the top predators. Unfortunately the locations of the paranasal sinus ostia are not top in the list of favorable advantages. Hemorrhoids, chronic back pain, varicous veins, etc are other morbid consequences of bipedalism.

\section{The Nose}

The top function of the nose going back from the fishes and up to the present is to smell for food and to avoid enemies. The paranasal sinuses and other structures are there to make this function happen; the paranasal sinuses have their own additional benefits to give:

- $\quad$ Lighten the skull

- Produce lysozyme to combat infection

- Source of mucus to trap bacteria and provide the conveyor belt with the cilia

- $\quad$ Resonator: singing and vocalization are enhanced

\section{Anatomy and bio-mechanics of Paranasal sinuses}

The paranasal sinuses come from the bulging of nasal pits into the bones that make up the nasal structure. The pits dilate into sacs which give rise to the frontal, the ethmoids, the sphenoids and the maxillary sinuses. Twenty million years ago or further into the past the location of the respective sinus ostia are favorably located for gravitational drainage but as we evolved to bipedalism that advantage is lost or diminished. (Figure 1)

The varying attitudes of hanging from tree branch or to walk on all fours favor drainage of the sinuses so we may

Mariano B. Caparas, MD

Department of Otorhinolaryngology

2nd Floor, Ward 10

Philippine General Hospital

University of the Philippines Manila

Taft Avenue, Ermita, Manila 1000 Philippines

Telephone: +632 5548400 local 2151 or 2152

Email: juvybaltazar@yahoo.com suspect that rhinosinusitis are not much of a health concern when we humans were still not hominids. (Figure 2)

\section{Frontal Sinus}

The usual shape of frontal sinus is like the ordinary kitchen funnel which easily drains by gravity alone; a little problematic are the well-developed types; fortunately they are rare where the overhang may lead to mucus stagnation and focus of infection. (Figure 3)

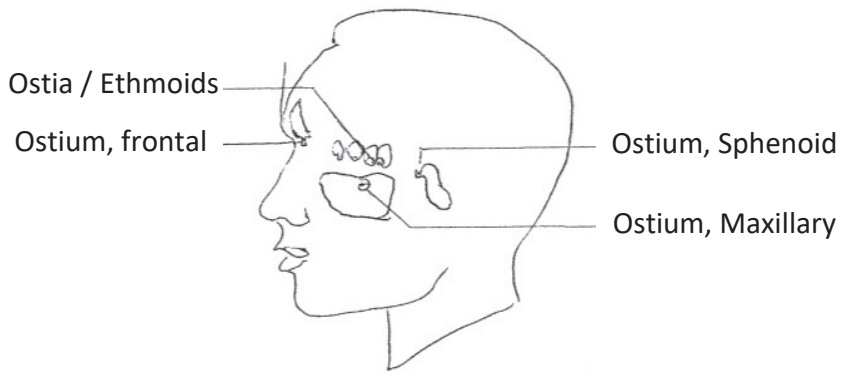

Figure 1.

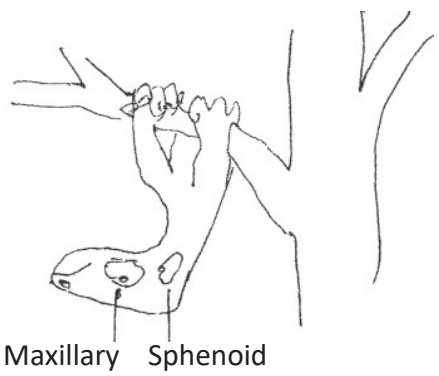

Figure 2.

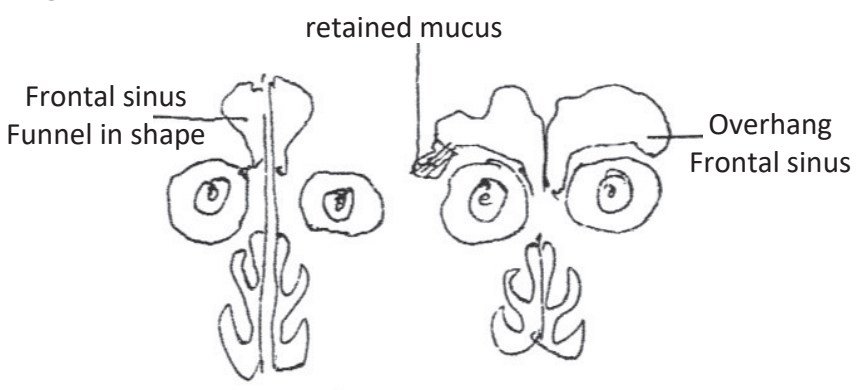

Figure 3.

Consequently, the incidence of clinical frontal sinusitis is not high; the occasional pyocele or mucocele is due to compression of the elongated duct as it traverses the ethmoid space; a nasal polyp or an enlarged ethmoid air cell may obstruct the flow. 


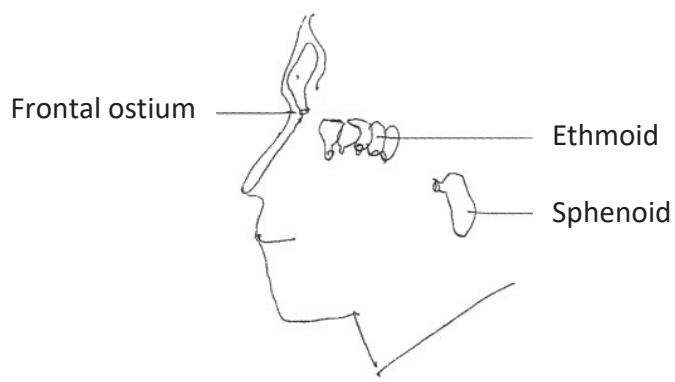

Figure 4.

\section{Ethmoid Air Cells}

On the average, there are eight to ten cells on each side, counting both the anterior and posterior group. Bipedalism and the nature of our work which is mostly sitting up favors drainage by gravity; the ethmoid cells may be easily affected by noxious agents because of the exposed location but relief is seen faster for the same reason. The Ethmoid given a chance to drain by itself will get well without much fuss. (Figure 4)

\section{Sphenoids}

The sphenoid sinuses are absent at birth but are fully developed by adulthood. In our normal erect position at work or at play, the natural ostium is located high in relation to the bottom of the sac especially when the sinuses are welldeveloped. Small sphenoid sac, even when infected heals fast and well; however the incidence of persistent or recurrent cases are seen in well-developed sphenoids.

Sphenoiditis is not as rare as is claimed in the journals, text books and by esteemed lecturers. Pressurized irrigation thru the ostium is the only reliable treatment and may be performed in regular intervals. Sphenoiditis I believe is the main culprit in chronic atrophic rhinitis and usually overlooked by the ENT specialist. The sphenoid sinus becomes a catch basin for the discharge coming from the frontal, ethmoids and maxillary sinuses if the person is in prone position, as in sleeping. This should explain in my mind why sphenoiditis is not an infrequent occurrence.

\section{Maxillary Sinus}

This is the most frequently encountered recalcitrant infection of the four paired paranasal sinuses for two reasons:

- Has a bigger volume - 8 to $15 \mathrm{cc}$

- The ostium or ostia are located high up in the wall in bipedal attitude. A dysfunctional ciliary belt has to work hard to dispose a greater amount of mucus working mostly against gravity

The accessory ostium plays a vital function acting as a relief valve or peep hole to equalize intrasinus air pressure in case the main ostium is blocked: like the extra hole in a can of evaporated milk which we are trying to empty. Drainage by the Bernouilli effect is improved if air could rush into the sinuses thru the accessory ostium. The Bernouilli mechanism is the partial vacuum around the ostium as the air rushes out as we blow our nose. (Figure 5)

Surgical antrostomy at the inferior meatus has been maligned unfairly in my opinion but it offers some advantages for recurrent maxillary sinusitis namely:

- Irrigating the maxillary sinuses of thick mucus is more efficiently and painlessly done thru the antrostomy

- If the accessory ostium is blocked or absent the surgical antrostomy acts as a relief opening thereby relieving the negative pressure in the sinus

This thought experiment may help the ENT specialist understand the physiopathology of the diseases of the paranasal sinuses, or at least provoke meaningful discussion.

\section{Epilogue}

Evolution marches on relentlessly and in so many million years the paranasal sinus ostia may be found in the place where gravity can work its wonder and make the life a little easier for future Homo Sapiens.

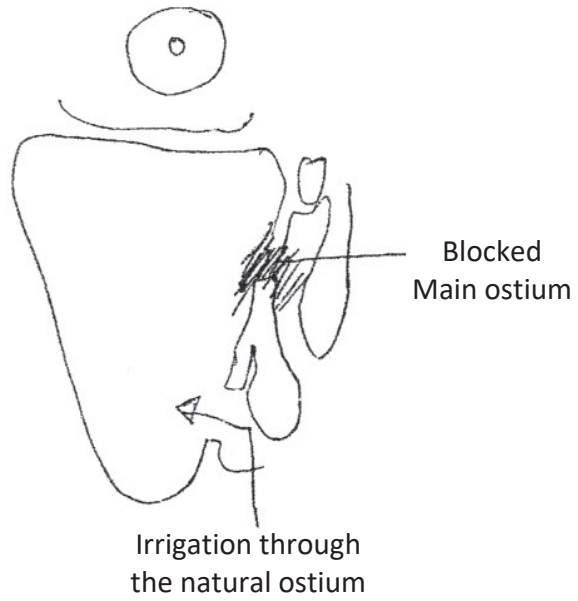

Figure 5.

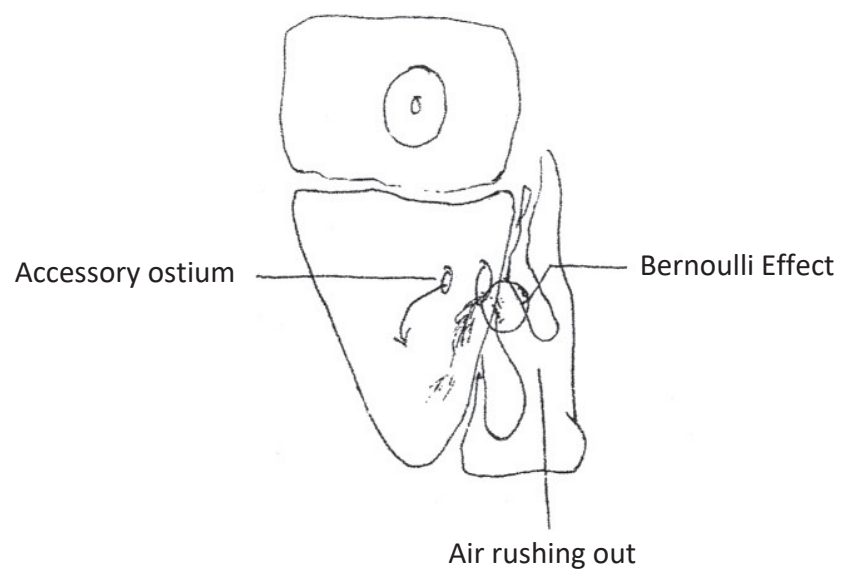

Air rushing out 